\title{
Geographical structure and differential natural selection among North European populations
}

\author{
Brian P. McEvoy, ${ }^{1,17}$ Grant W. Montgomery, ${ }^{1}$ Allan F. McRae, ${ }^{1}$ Samuli Ripatti, ${ }^{2,3,4}$ \\ Markus Perola, ${ }^{2,3}$ Tim D. Spector, ${ }^{5}$ Lynn Cherkas, ${ }^{5}$ Kourosh R. Ahmadi, ${ }^{5}$ \\ Dorret Boomsma, ${ }^{6}$ Gonneke Willemsen, ${ }^{6}$ Jouke J. Hottenga, ${ }^{6}$ Nancy L. Pedersen, ${ }^{4}$ \\ Patrik K.E. Magnusson, ${ }^{4}$ Kirsten Ohm Kyvik, ${ }^{7,8}$ Kaare Christensen, ${ }^{7}$ Jaakko Kaprio, ${ }^{3,9,10}$ \\ Kauko Heikkilä, ${ }^{9}$ Aarno Palotie, ${ }^{3,14,15,16}$ Elisabeth Widen, ${ }^{3}$ Juha Muilu, ${ }^{3}$ \\ Ann-Christine Syvänen, ${ }^{11}$ Ulrika Liljedahl, ${ }^{11}$ Orla Hardiman, ${ }^{12}$ Simon Cronin, ${ }^{13}$ \\ Leena Peltonen, ${ }^{2,3,14,15}$ Nicholas G. Martin, ${ }^{1}$ and Peter M. Visscher ${ }^{1}$
}

${ }^{1}$ Queensland Institute of Medical Research, Brisbane, Queensland 4029, Australia; ${ }^{2}$ National Institute for Health and Welfare (THL), Helsinki FI-00271, Finland; ${ }^{3}$ Institute for Molecular Medicine Finland (FIMM), University of Helsinki, Helsinki FI-00014, Finland; ${ }^{4}$ Department of Medical Epidemiology and Biostatistics, Karolinska Institutet, Stockholm SE-171 77, Sweden; ${ }^{5}$ Department of Twin Research and Genetic Epidemiology, King's College London, St. Thomas' Hospital Campus, London SE1 7EH, United Kingdom; ${ }^{6}$ Department of Biological Psychology, Vrije Universiteit, Amsterdam 1081 BT, The Netherlands; ${ }^{7}$ Department of Epidemiology, Institute of Public Health, University of Southern Denmark, Odense DK-5000, Denmark; ${ }^{8}$ Institute of Regional Health Services Research, University of Southern Denmark, Odense DK-5000, Denmark; ${ }^{9}$ Faculty of Medicine, Department of Public Health, University of Helsinki, Helsinki Fl-00014, Finland; ${ }^{10}$ Department of Mental Health, National Institute for Health and Welfare (THL), Helsinki FI-00271, Finland; ${ }^{11}$ Department of Medical Sciences, Uppsala University, Uppsala 75185, Sweden; ${ }^{12}$ Trinity College Institute of Neuroscience, Trinity College, Dublin 2, Ireland; ${ }^{13}$ Department of Clinical Neurological Sciences, Royal College of Surgeons in Ireland, Dublin 2, Ireland; ${ }^{14}$ The Broad Institute, Massachusetts Institute of Technology, Cambridge, Massachusetts 02142, USA;

${ }^{15}$ Wellcome Trust Sanger Institute-Wellcome Trust Genome Campus, Hinxton, Cambridge CB10 1HH, United Kingdom;

${ }^{16}$ Department of Clinical Chemistry, University of Helsinki, Helsinki FI-00014, Finland.

Population structure can provide novel insight into the human past, and recognizing and correcting for such stratification is a practical concern in gene mapping by many association methodologies. We investigate these patterns, primarily through principal component (PC) analysis of whole genome SNP polymorphism, in 2099 individuals from populations of Northern European origin (Ireland, United Kingdom, Netherlands, Denmark, Sweden, Finland, Australia, and HapMap EuropeanAmerican). The major trends ( $\mathrm{PCl}$ and PC2) demonstrate an ability to detect geographic substructure, even over a small area like the British Isles, and this information can then be applied to finely dissect the ancestry of the European-Australian and European-American samples. They simultaneously point to the importance of considering population stratification in what might be considered a small homogeneous region. There is evidence from $F_{\mathrm{ST}}$-based analysis of genic and nongenic SNPs that differential positive selection has operated across these populations despite their short divergence time and relatively similar geographic and environmental range. The pressure appears to have been focused on genes involved in immunity, perhaps reflecting response to infectious disease epidemic. Such an event may explain a striking selective sweep centered on the rs2508049-G allele, close to the HLA-G gene on chromosome 6. Evidence of the sweep extends over a 8-Mb/3.5-cM region. Overall, the results illustrate the power of dense genotype and sample data to explore regional population variation, the events that have crafted it, and their implications in both explaining disease prevalence and mapping these genes by association.

[Supplemental material is available online at www.genome.org. The genotype data from this study have been submitted to the European Genotype Archive (http://www.ebi.ac.uk/ega/page.php), under accession no. EGASO0000000033.]

Patterns of genetic variation within and between human populations have long provided novel insights into the origin and history of different groups. The advent of whole genome association (WGA) mapping has also highlighted the practical importance of identifying and understanding these patterns. A mismatch in the ancestry of individuals in a simple case/control

${ }^{17}$ Corresponding author.

E-mail brian.mcevoy@qimr.edu.au; fax 61-7-3362-0101.

Article is online at http://www.genome.org/cgi/doi/10.1101/gr.083394.108. association paradigm can lead to false positives and/or reduced power to detect associations.

Studies of population-level whole genome (WG) polymorphism were initially restricted to the International HapMap populations (Yoruban, Japanese, Chinese, and European-Americans) but provided valuable information on intercontinental variation across the human genome, including structural variation, recombination, and selection (International HapMap Consortium $2005,2007)$. The whole genome approach has now begun to be applied to more nuanced intracontinental variation within 
Europe. First generation studies using European-Americans or small numbers of in situ Europeans (but with relatively few markers) quickly identified a clear North-South split in the continent's population and hinted at further structure (Seldin et al. 2006; Bauchet et al. 2007; Price et al. 2008; Seldin and Price 2008; Tian et al. 2008). Analysis of WG variation in larger numbers of individuals sampled in situ from multiple European populations has recently extended these findings. Using principal component analysis (PCA) of up to $\approx 300,000$ SNPs, they have shown a remarkable correlation of an individual's position in genetic space to their geographic origin (Heath et al. 2008; Lao et al. 2008; Novembre et al. 2008).

We continue this progression by exploring subcontinental WG (300K) variation in 2099 individuals from six Northern European populations (Ireland, United Kingdom, Netherlands, Sweden, Denmark, and Finland), as well as two descendent New World populations (European-Australians and the European-American HapMap sample). The data demonstrate and confirm an ability to dissect regional to subregional geographic structure and also point to the discernable impact of differential natural selection on the recently diverged Northern European populations. Both of these observations have important present-day consequences in explaining disease incidence and in mapping complex traits through association methods.

\section{Results}

\section{Principal component analysis of Northern Europeans}

We initially investigated the genetic structure of eight European population samples (Netherlands, Sweden, Denmark, Ireland, United Kingdom, Finland, HapMap European-American [CEU], and Australia) using principal component analysis (PCA) of 296,553 autosomal SNPs. We did not prune the genotypes with respect to linkage disequilibrium (LD) prior to PCA since this allows us to investigate both population substructure and large genomic structural/haplotypic variation. The novel genotype data from this study are deposited at the European Genotype Archive (see Methods).

The top 100 PCs were generated using the EIGENSOFT package (Patterson et al. 2006; Price et al. 2006); however, we focused on the top five since the eigenvalues remain relatively constant in subsequent PCs (Supplemental Fig. S1). PC1 and PC2 are plotted together in Figure 1A. Individuals from the different populations are largely separable, although there is clearly some overlap, suggesting that variation is generally continuous rather than discrete. Mantel testing (Mantel 1967) confirms that differences in individual PC1 or PC2 scores are strongly correlated with the geographic distance between sampled individuals $(r=0.76, P<$ 0.0001 and $r=0.36, P<0.0001$, respectively). PC1 most obviously separates the Finnish individuals from the other Northern Europeans and, more subtly, these from each other. Remarkably, given the smaller geographic distances, this pattern is apparent even within Finland once regional origins are considered (Supplemental Fig. S2). PC2 tends to separate the insular Irish and U.K. populations from each other and from their closest continental neighbors. We repeated PCA excluding the Finns, given their relatively outlying positions, and were still able to discern good separation between the remaining populations with the originally observed PC2 becoming the most prominent pattern in the data (PC1 in Supplemental Fig. S3).
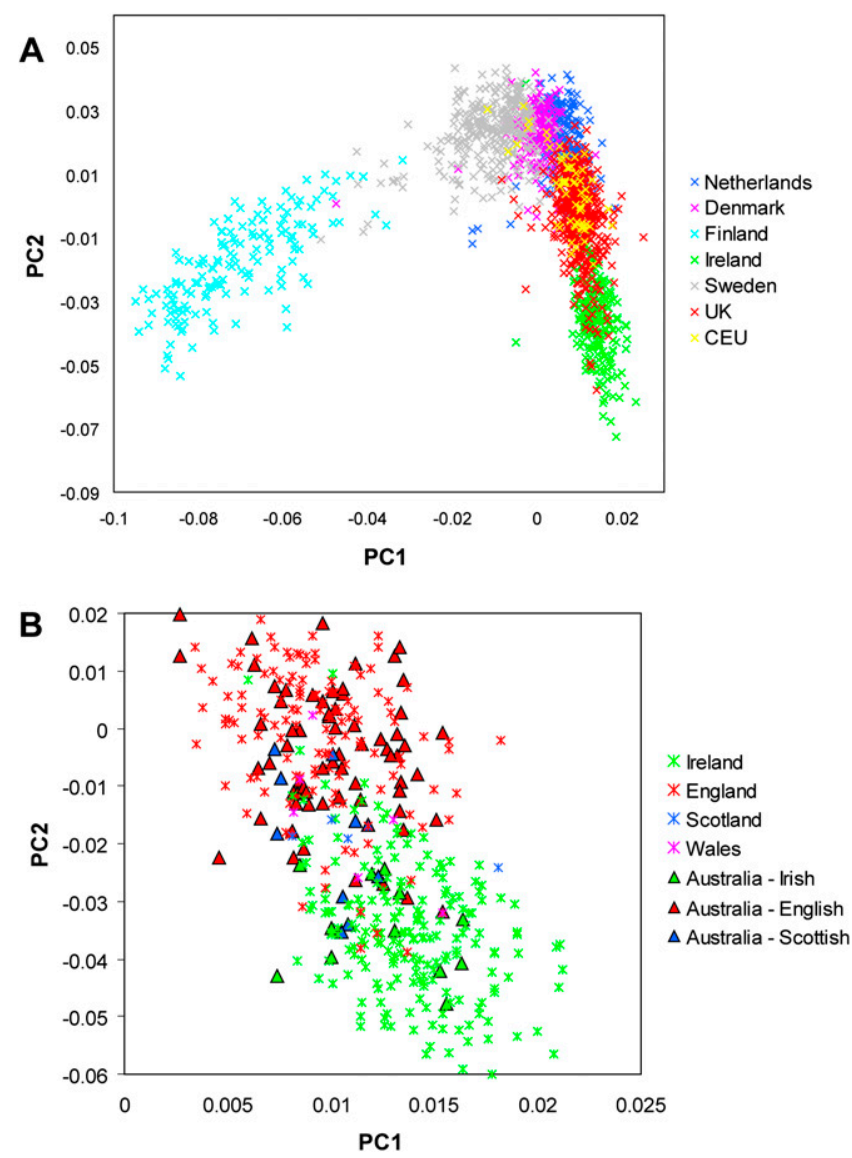

Figure 1. PCA of Northern European population structure. (A) PC1 versus PC2 from 2051 individuals genotyped for 296,553 autosomal SNPs. PCA was conducted including the Australian sample $(n=451)$, but these are not shown here (see Supplemental Fig. S4). (B) PC1 versus PC2 focused on the United Kingdom, Irish, and Australian populations. For ease of illustration, PC1 has been constrained to between 0.02 and -0.06 and PC2 to between 0 and 0.025 . Only those U.K. samples with birthplace information are displayed $(n=143)$, and these are distinguished as England, Scotland, and Wales. Australian samples whose four grandparental ancestries are from one country are also shown.

As previous studies have noted (Bauchet et al. 2007; Lao et al. 2008), the position of CEU HapMap individuals in the genetic space is consistent with their putative Northwest European ancestry. This illustrates the potential for ancestry reconstruction given sufficient numbers of markers and appropriate comparative populations, an application we used next to investigate the origin of a large Australian population sample.

\section{Population structure of Australia and the British Isles}

Approximately $85 \%$ of current Australians are descendents of European settlers who began arriving in 1788 . The vast majority of these originated in Britain or Ireland but were joined by smaller numbers of Germans, Greeks, Italians, and Eastern Europeans (among others) in the 19th and 20th Centuries.

Fourteen individuals from our Australian sample $(n=465)$ were identified as outliers in eigenvector analysis, and subsequent checks revealed that these had self-reported non-European or Southern European ancestry. Virtually all of the remaining Australians are distributed in the PC1-PC2 regions occupied by the 
United Kingdom (noting its overlap with the Dutch sample) and Irish populations (Fig. 1B; Supplemental Fig. S4). PC2 is most informative at distinguishing the United Kingdom and Irish populations. The mean Australian PC2 value $(-0.01)$ is closer to the United Kingdom $(-0.003)$ than Ireland $(-0.036)$, with the difference between Australia and the United Kingdom $\sim 22 \%$ of the total distance between the United Kingdom and Irish means along this axis. Interestingly, this figure coincides with the fraction of all British Isles self-reported ancestry mentions that are Irish (Source: 2006 Census, Australia Bureau of Statistics; http:// www.abs.gov.au/).

Several previous studies, using single marker systems and most recently whole genome SNP data (Wellcome Trust Case Control Consortium 2007), have noted a gradient of genetic variation running approximately Southeast to Northwest across Britain or the British Isles. Although we did not have detailed regional ancestry information for the United Kingdom or Irish samples, we used place of birth, which was available for a subset of the United Kingdom sample $(n=143)$ to divide these into English $(n=133)$, Scottish $(n=4)$, and Welsh $(n=6)$ cohorts. Although numbers are small, the mean PC2 values for the Scottish and Welsh cohorts are intermediate between those of England and Ireland (Fig. 1B).

We also availed of detailed self-reported ancestry for the Australian sample to distinguish three further cohorts whose fourgrandparental ancestries are English $(n=61)$, Irish $(n=12)$, or Scottish $(n=10)$. There is a significant correspondence of this selfreported ancestry with PC2 scores (one-way ANOVA, $P<0.0001$ ). Once again the average intermediate position of the AustralianScots (mean PC2 score of -0.019 vs. -0.005 in the English and -0.035 in Irish-Australian cohorts) is evidence that further subregional patterns can be discerned across the British Isles (Fig. 1B).

\section{Ancestry informative markers and structure}

We next explored whether smaller numbers of SNPs, which are most differentiated between our populations, are effective at capturing the variation revealed by the full $296 \mathrm{~K}$ markers. Panels of Ancestry Informative Markers (AIMs) could have important practical applications in detecting and correcting for variation in individual ancestry that can confound some association gene mapping methods by increasing false positive results and/or reducing power. This is particularly the case in candidate genedriven studies or follow-up replication attempts where a full suite of genome polymorphism is not available.

The Northern European sample population was divided in half to give a discovery $(n=770)$ and a test panel $(n=1281$, which included Australia and CEU). $F_{\mathrm{ST}}$ values between populations in the discovery panel were used to select 500, 1500, 2500, and 5000 top-ranked SNP markers as AIM sets with the additional condition that no marker was within $250 \mathrm{~kb}$ of another, a measure taken to minimize LD between SNPs. The performance of these AIM panels was then assessed in the test data set. All panels returned PC1 scores for individuals that were significantly correlated to the values revealed by the full data set, both when the Finns were included and excluded ( $r \geq 0.91$ and $r \geq 0.67$, respectively, with minimum $r$ values observed using the 500 SNP sets). All sets performed substantially better than random marker sets of the same size especially as AIM panel size decreased (Supplemental Fig. 5A). In a similar analysis of PC2, the 5000 AIM set preserved a moderate correlation $(r=0.649, P<0.05)$ to the full SNP test set, but further reductions in AIM number led to a steep decline in correlation, and they are no longer significant for sets of 1500 or 500 markers either $F_{\mathrm{ST}}$-based or randomly selected (Supplemental Fig. 5A).

We also explored the performance of the AIM panels using an alternative Bayesian modeling approach implemented in the structure package (Pritchard et al. 2000; Falush et al. 2003). We examined each AIM panel over $K=2$ to $K=5$ in the test sample set, where $K$ is the predefined number of populations into which the data are to be split (Supplemental Figs. 5B and 6). At $K=2$, all AIM panels were able to distinguish the Finnish samples from other populations. At $K=3$, there was little obvious coherent division of the remaining European samples for AIM sets of 500 to 2500 SNPs. However, at 5000 markers, some distinction between the British Isles (especially the Irish) and non-Finnish continental samples became apparent (Supplemental Fig. 5B). This is reminiscent of $\mathrm{PC} 2$, and the results again appear to demonstrate the majority British Isles ancestry of the Australian sample. We also repeated the AIM selection and structure analysis for 5000 SNPs excluding the Finnish sample and discerned some tendency for the separation of the British Isles (especially Irish) from the continental Europeans over various $K$ values (Supplemental Fig. 5C). Overall, the results illustrate the potential to develop a single AIM set that will be informative on even subtle Northern European variation, an ability that will increase as bigger sets of initially genotyped markers (in more populations) become available. (A list of the top 10,000 SNPs by $F_{\mathrm{ST}}$ value is given in Supplemental Table 1.

\section{Extent of population stratification}

Mismatched ancestry between cases and control groupings in a standard whole genome association study is a potential source of Type 1 and 2 errors. However, balanced against this concern are the potentials to increase power by combining (often rare) cases across populations and achieve significant cost savings by reusing standard sets of controls. The genomic control inflation factor $\left(\lambda_{\mathrm{gc}}\right)$ is one way to quantify the impact of population stratification between cases and controls (Devlin and Roeder 1999). However, this is highly dependent on sample sizes as well as the level of ancestry mismatch between groups as measured by $F_{\mathrm{ST}}$. As theory suggests (and as we empirically observe-see Supplemental Fig. 7), the relationship between $\lambda_{\mathrm{gc}}, F_{\mathrm{ST}}$, and total sample size ( $n$ individuals) is approximately $E\left(\lambda_{\mathrm{gc}}\right) \sim 1+\left(n * F_{\mathrm{ST}}\right)$ when $F_{\mathrm{ST}}$ is small (such as the levels between European populations) and the numbers of "cases" and "controls" are the same.

As an illustration of the potential impact of Northern European stratification, we calculated expected pairwise $\lambda_{\mathrm{gc}}$ values between populations (equivalent to a situation in which cases and controls come entirely from different populations) given the $F_{\mathrm{ST}}$ values in Table 1 and assuming a total sample size of 1000 individuals. These show that a random United Kingdom population sample is well matched to the Australians $\left(\lambda_{g c}=1.025\right)$ after obvious outliers are removed from both, either by self-reported ancestry or by EIGENSTRAT. However, other combinations show substantial inflation and corresponding potential for false positives (Table 1).

\section{Differential natural selection among Northern Europeans}

While the differentiation/structure observed between our populations is expected to be mainly a consequence of neutral evolution (drift) and demographic processes like migration, it is also possible that natural selection has played a role in shaping the diversity of some SNPs. One simple expected signature of 
Table 1. Population pairwise $F_{\mathrm{ST}}$ (above the diagonal) and expected $\lambda_{\mathrm{gc}}$ values (below the diagonal)

\begin{tabular}{|c|c|c|c|c|c|c|c|c|}
\hline Population & Australia & CEU-HapMap & Denmark & Finland & Ireland & Netherlands & Sweden & UK \\
\hline Australia & 0 & 0.00027 & 0.00056 & 0.00636 & 0.0004 & 0.00045 & 0.00098 & 0.00003 \\
\hline CEU-HapMap & 1.27 & 0 & 0.00046 & 0.00637 & 0.00092 & 0.00043 & 0.00086 & 0.00021 \\
\hline Denmark & 1.56 & 1.46 & 0 & 0.00551 & 0.00141 & 0.00036 & 0.00035 & 0.00045 \\
\hline Finland & 7.36 & 7.37 & 6.51 & 0 & 0.00721 & 0.00627 & 0.00437 & 0.00642 \\
\hline Ireland & 1.40 & 1.92 & 2.41 & 8.21 & 0 & 0.00131 & 0.00181 & 0.00055 \\
\hline Netherlands & 1.45 & 1.43 & 1.36 & 7.27 & 2.31 & 0 & 0.00082 & 0.00034 \\
\hline Sweden & 1.98 & 1.86 & 1.35 & 5.37 & 2.81 & 1.82 & 0 & 0.00091 \\
\hline UK & 1.03 & 1.21 & 1.45 & 7.42 & 1.55 & 1.34 & 1.91 & 0 \\
\hline
\end{tabular}

Expected $\lambda_{\mathrm{gc}}$ values based on a case-control experiment of 500 cases and 500 controls, using $E\left(\lambda_{\mathrm{gc}}\right)=1+1000 * F_{\mathrm{ST}}$.

differential positive selection-the action of a selective force in some populations but not others-is the overrepresentation of SNPs with high $F_{\mathrm{ST}}$ values in genic regions, since these are a (albeit imperfect) proxy for functional polymorphism. A recent study demonstrated this effect for an $F_{\mathrm{ST}}$ comparison between the HapMap populations (Barreiro et al. 2008).

We divided $F_{\text {ST }}$ values calculated at each autosomal SNP in our six in situ European populations (Denmark, Ireland, Netherlands, Finland, United Kingdom, and Sweden) into 10 bins of increasing (0.002 increment) $F_{\mathrm{ST}}$ floor and noted a significant excess of genic SNPs in the top $F_{\mathrm{ST}}$ $(>0.016)$ category $\left(\chi^{2}=7.4\right.$; d.f. $=1 ; P=$ 0.0071; Fig. 2A). The $\chi^{2}$ test may be nonconservative in these circumstances given the potential nonindependence of markers due to LD. However, this is mitigated to some extent by the "tagging" criteria inherent in the selection of SNPs for the genotyping platform. Furthermore, stringent pruning for LD would bias the genic/nongenic test against detecting selection since the "hitchhiking" effect is a key footprint of its action. The effect was still detectable but weaker after the Finnish population was removed $\left(\chi^{2}=4.9 ;\right.$ d.f. $\left.=1 ; P=0.028\right)$. However, given the relative genetic distinction of the Finnish, the absolute $F_{\mathrm{ST}}$ value for each SNP is generally lower after their exclusion, and only 50 SNPs exceed $F_{\text {ST }}$ of 0.016 (compared to 1358 including Finland). Rescaling to a cut-off of $F_{\mathrm{ST}}>0.01$ still preserves the enrichment trend $\left(\chi^{2}=4.0\right.$; d.f. $\left.=1 ; P=0.046\right)$.

Interpretation of these findings requires a consideration of any ascertainment bias underlying the inclusion of genic versus nongenic SNPs on the genotyping platform and whether any systematic bias in the relationship of minor allele frequency (MAF) to $F_{\mathrm{ST}}$ exists. The overall genic/nongenic distribution of SNPs is very weakly but significantly different (Kolmogorov-Smirnov test: $D=$ $0.0117, P=7.5 \times 10^{-9}$; Supplemental Fig. S8A) owing to a slight excess of genic SNPs in the lower MAF categories. We therefore randomly sampled from the genomic position. nongenic SNPs to ensure that the number in each MAF bin was exactly equal (even though this involves discarding $\approx 20 \%$ of the SNPs and potentially reducing the power to detect any effect). Based on 1000 such replicate analyses, we continue to observe a similar trend of genic enrichment in relation to high $F_{\mathrm{ST}}(P=$ 0.017). Furthermore, the average $F_{\mathrm{ST}}$ estimate was similar across most $F_{\mathrm{ST}}$ categories, suggesting that difference in MAF could not explain the finding (Supplemental Fig. S8B). Indeed, there is some evidence of systematically lower $F_{\mathrm{ST}}$ values in lower MAF categories, which would bias against detecting the observed excess
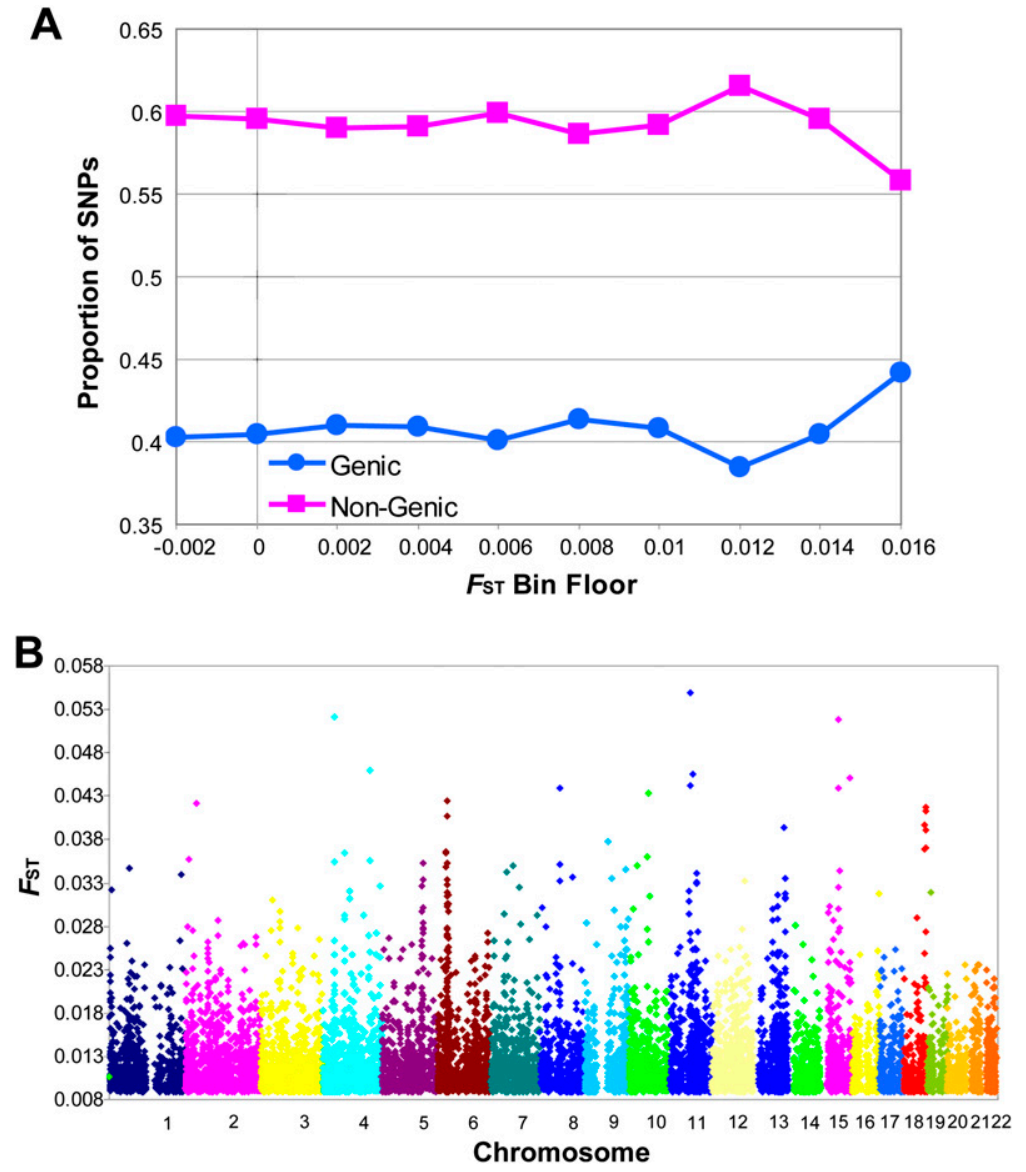

Figure 2. Northern European $F_{\mathrm{ST}}$ values by genomic location. $(A)$ Proportion of SNPs that are inside (genic) or outside (nongenic) genes by $F_{S T}$ bin category. $X$-axis $F_{S T}$ values refer to the lower boundary of the bin. $(B)$ Genome-wide distribution of the top 10,000 $F_{\text {ST }}(>0.00888)$ values by chromosome and 
(since genic SNPs are somewhat overrepresented in these lower categories).

The genomic locations of the top 10,000 SNPs by $F_{\mathrm{ST}}$ value are shown in Figure 2B (with a more detailed view available in Supplemental Table 1). There is clustering of high $F_{\mathrm{ST}}$ values at certain locations, and, while it is impossible to definitively ascribe any particular region of high $F_{\mathrm{ST}}$ values to positive selection, these may be considered candidate regions for its action. We identified the most prominent of these (which had a peak $F_{\mathrm{ST}}$ within the top 100 ranked values or $F_{\mathrm{ST}}>\approx 0.028$; Table 2 ). Consistent with similar analysis in the United Kingdom population (Wellcome Trust Case Control Consortium 2007), the human-leukocyte-antigen (HLA) region of chromosome 6 is the most obvious candidate. However, the enrichment of genic SNPs in the top $F_{\mathrm{ST}}$ category remains significant $\left(\chi^{2}=6.2\right.$; d.f. $\left.=1 ; P=0.011\right)$ even when a large region around the top HLA SNP is removed from the analysis. There is also a prominent peak on chromosome 5 (around rs9784675/position $132.1 \mathrm{Mb}$ ). Several genes in this area are, like many of those in the HLA region, involved in immune response including several interleukins and their receptors. A further peak on chromosome 4 is close to several Toll-like receptors and was also identified as highly differentiated between United Kingdom regions (Wellcome Trust Case Control Consortium 2007). Guided by these anecdotal observations, we tested whether SNPs in the highest $F_{\mathrm{ST}}$ category $(>0.016)$ were overrepresented among genes of a particular functional class by using the PANTHER gene ontology database (Thomas et al. 2003). The "Immunity and Defense" (BP00148) category is the only one of the 23 ontological terms (with sufficient numbers to be tested) to show a significant enrichment of high $F_{\mathrm{ST}}$ SNPs after correction for multiple testing $(P=0.0012$, adjusted significance level $=0.0022$ ). As a contrast, we examined the ontology of the 1358 SNPs with the lowest $F_{\mathrm{ST}}$ values $\left(F_{\mathrm{ST}} \approx 0\right)$ and found that no category was close to either nominal or multiple test adjusted significance.

While this suggests that the selective signal is focused on immune function, it does not preclude the action of differential selection at some other individual loci. For example, the distribution of top $F_{\text {ST }}$ points to one cluster of high values centered on the HERC2 gene on chromosome 15. HERC2 is close to OCA2, which substantially controls eye color (Sturm et al. 2008) and has been identified in several studies as a selection target in Europeans (Voight et al. 2006). Several additional peak SNPs do not fall within $250 \mathrm{~kb}$ of known (or validated) genes. These may simply be poorly localized by the peak SNP, false positive signatures of selection, or a sign that remote regulatory elements/and or other noncoding DNAs are also selective targets. The lactase gene (LCT) on chromosome 2 , which is a well-established target of selection, is not prominent in this survey, an observation probably explained by a similar strength of selection across Northern Europe.

\section{PC3 and PC4 reflect large-scale genome structural variation}

PC5 displays a significant correlation with geographic distance, although it is substantially weaker (Mantel test: $r=0.067 ; P<$ 0.0001) than those observed with PC1 and PC2. Accordingly, the geographic patterning is less obvious and defies simple description (Supplemental Figs. S3 and S9). It appears to somewhat separate Ireland and Sweden from the remaining populations. The explanation for this is unclear although it does not seem to be a genotyping artifact since individual PC5 scores are not correlated with missing data, nor is the observation consistent with any potential batch effect. Further PCs (6-10) were found not to correlate with geography.

Interestingly, PC3 and PC4 were also not stratified by population label, but, rather, PC4 shows a neat three-way split of individuals, a pattern reminiscent of that seen in the third PC of a similarly genotyped European-American data set (Fig. 3A; Tian et al. 2008). This reflected the division of individuals based on the three possible genotypes of a large $(\approx 4 \mathrm{Mb})$, polymorphic inversion on chromosome 8p23.1 (between 8 and $12 \mathrm{Mb}$ ). We confirmed that the same feature was responsible for our PC4 by dividing individuals into two extreme cohorts based on their PC4 score $(<-0.02, n=385$ and $>0.01, n=647)$ and calculating the allele frequency difference $(\delta)$ between groups for each SNP. The top 554 SNPs localize to chromosome 8 between 8.135 and $11.90 \mathrm{Mb}$.

PC3 also appears to split individuals, regardless of population origin, into at least two groups. Reasoning that it might also reflect

Table 2. Genomic regions showing high $F_{\mathrm{ST}}$ clustering

\begin{tabular}{|c|c|c|c|c|}
\hline Peak SNPa & Peak $F_{\mathrm{ST}}{ }^{\mathrm{a}}$ & Chromosome & Position $^{\mathbf{b}}$ & Genes $^{c}$ \\
\hline rs1388612 & 0.0298 & 3 & 62219484 & PTPRG C3orf14, CADPS \\
\hline rs878456 & 0.052 & 4 & 38208152 & KLF3, TLR10 \\
\hline rs2088092 & 0.0365 & 4 & 70333310 & UGT2B11, UGT2B28, UGT2B4, UGT2A1 \\
\hline rs 9784675 & 0.036 & 5 & 132097638 & $\begin{array}{l}\text { KIF3A, IRF1, IL5, RAD50, IL13, IL4, CCNI2, } \\
\quad \text { ANKRD43, SHROOM1, GDF9, UQCRQ, AFF4, LEAP2 }\end{array}$ \\
\hline rs2071593 & 0.0406 & 6 & 31620778 & ATP6V1G2, HLA ${ }^{\mathrm{d}}$ \\
\hline rs4738873 & 0.0439 & 8 & 62248730 & RLBP1L1 \\
\hline rs3739555 & 0.0345 & 9 & 128980237 & RALGPS1, ANGPTL2, GARNL3, SLC2A8, ZNF79 \\
\hline rs1454027 & 0.0341 & 11 & 83565887 & DLG2 \\
\hline rs9563972 & 0.03 & 13 & 62207209 & - \\
\hline rs1667394 & 0.0303 & 15 & 26203777 & HERC2, OCA2 \\
\hline rs4075612 & 0.0418 & 18 & 69103200 & - \\
\hline
\end{tabular}

${ }^{\text {a }}$ Regions were identified from a visual inspection of a plot of the top 10,000 $F_{\mathrm{ST}}$ values against genomic position. Reported SNP and $F_{\mathrm{ST}}$ values are the highest observed in that cluster.

${ }^{b} \mathrm{NCBI} 36$ build of the human genome.

'Genes within $250 \mathrm{~kb}$ either side of the peak SNP are listed. Where the SNP falls within a gene, the gene symbol is indicated in bold.

${ }^{\mathrm{d}} \mathrm{A}$ further 59 genes within this region are in proximity $( \pm 250 \mathrm{~kb})$ to the peak SNP: AIF1, APOM, ATP6V1 G2, BAT1, BAT2, BAT3, BAT4, BAT5, C2, C4A, C4B, C6orf21, C6orf25, C6orf26, C6orf27, C6orf47, C6orf48, CCHCR1, CDSN, CFB, CLIC1, CSNK2B, CYP21A2, DDAH2, DOM3Z, EHMT2, HCG27, HCP5, HLA-B, HLA-C, HSPA1A, HSPA1B, HSPA1L, LSM2, LST1, LTA, LTB, LY6G5B, LY6G5C, LY6G6C, LY6G6D, MCCD1, MICA, MICB, MSH5, NCR3, NEU1, NFKBIL1, POU5F1, PSORS1C2, RDBP, SKIV2L, SLC44A4, STK19, TCF19, TNF, TNXB, VARS, ZBTB12. 

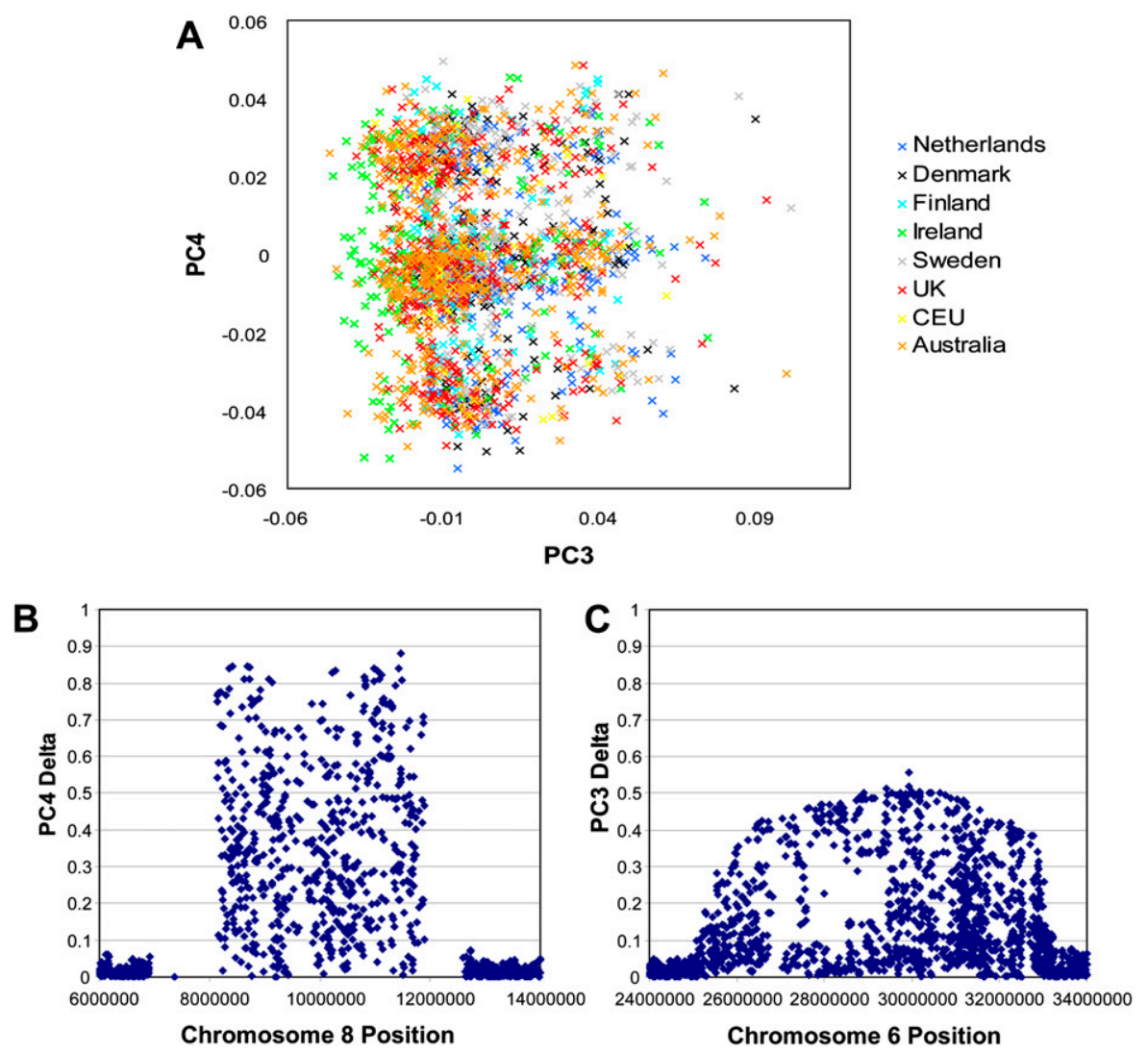

Figure 3. $\mathrm{PC} 3$ and PC4 in Northern European populations. $(A)$ PC 3 versus PC4 derived from 2051 individuals genotyped for 296,553 autosomal SNPs. (B) An 8-Mb section of chromosome 8 (6 Mb to 14 $\mathrm{Mb}$ ) showing the distribution of SNP $\delta$ values derived from extreme PC4 cohorts. (C) A 10-Mb section of chromosome $6(24 \mathrm{Mb}$ to $34 \mathrm{Mb}$ ) showing the distribution of SNP $\delta$ values derived from extreme PC3 cohorts. Whereas $\delta$ values remain high over the entire chromosome 8 inversion region ( $8 \mathrm{Mb}$ to $12 \mathrm{Mb}$ ), those on chromosome 6 show a gradual decay upstream and downstream from the peak $\delta$ value observed at rs2508049 (position $29.99 \mathrm{Mb}$ ).

large structural variation, we divided samples into two extreme PC3 score cohorts (high: $>0.02, n=376$ and low: $<0, n=1256$ ) and again calculated $\delta$ for each autosomal SNP. Of the top $1000 \delta$ values, 975 fall within an 8-Mb region of chromosome 6 (25.1 Mb$33.2 \mathrm{Mb}$ ) encompassing the HLA region. However, the distribution of values is distinct from that observed on chromosome 8 (Fig. $3 \mathrm{~B}, \mathrm{C})$. The highest $\delta$ values in the chromosome 8 region are distributed across the $4-\mathrm{Mb}$ block consistent with a discrete inversion structure. Those in the chromosome 6 region, however, display a marked decrease both $5^{\prime}$ and $3^{\prime}$ of the maximum $\delta$ value observed at the rs2508049 SNP (position 29931862), consistent with a large haplotype decaying with increased recombination distance from this core.

There is a strong association between rs2508049 genotype and PC3 scores (ANOVA, $P=3.2 \times 10^{-293}$; Supplemental Fig. S10) with copy number of the $G$ allele (present at a frequency of $18.3 \%$ in our entire population sample) correlated with higher PC3 scores. Deleting SNPs from a 20-Mb region of chromosome 6 (20 $\mathrm{Mb}$ to $40 \mathrm{Mb}$ ) abolishes PC3 as originally observed.

\section{Selective sweep around rs2508049 in Northern Europeans}

We investigated the structure of this region further by phasing a $20-\mathrm{Mb}$ segment of chromosome 6 centered on rs 2508049 (6p22.3-21.2 from $20 \mathrm{Mb}$ to $40 \mathrm{Mb}$, encompassing 3080 SNPs), followed by extended haplotype homozygosity $(\mathrm{EHH})$ analysis. EHH measures the probability that two haplotypes with the same core allele (in our case, either $\mathrm{G}$ or A at rs2508049) will be identical to a defined distance or particular SNP (Sabeti et al. 2002). Figure 4A shows $\mathrm{EHH}$ scores for each core allele across a 5-cM $(9-\mathrm{Mb})$ region of chromosome 6 for all the Northern European populations as well as the African (YRI) and Asian (ASN) HapMap populations. The rs2508049-G core shows substantially higher levels of $\mathrm{EHH}$ than its sister rs2508049-A allele in all European samples over a region spanning $\sim 3.5 \mathrm{cM} / 8 \mathrm{Mb}$. While European populations and the African and Asian samples share a similar rs2508049-A EHH pattern, the low frequency of the $\mathrm{G}$ allele in the Asian population $(1 \%$, or two chromosomes out of 178) and its absence in the West African Yoruba sample preclude any meaningful analysis of the rs2508049-G EHH in these populations.

To determine whether the structure around rs2508049 is unusual in the Northern European genome, we used an empirical "significance" approach using the pre-phased chromosome $6 \mathrm{CEU}$ HapMap data (see Methods). A total of 15,325 SNP alleles were identified with a similar frequency $( \pm 2.5 \%)$ to the rs2052089-G allele (18.3\%). The empirical distributions of the 30,650 $\mathrm{EHH}$ observations (an upstream and downstream value for each SNP), over a range of distances from the cores, were used to construct a $95 \%$ boundary curve. The EHH pattern around rs2508049 in these data is similar to that observed in the other European, including the CEU, populations that were phased separately (Fig. 4B). Furthermore, the EHH values of the rs2508049-G allele run substantially ahead of the empirically derived 95 percentile over a 2 -cM region $3^{\prime}$ of the core SNP. The 5' pattern is more complex with rs2508049-G EHH values running consistently ahead up to $0.167 \mathrm{cM}$ from the core and then again from $0.34 \mathrm{cM}$ to $1.1 \mathrm{cM}$. In the interval it is close to the 95th percentile, criss-crossing slightly above and under at various distances. However, the overall pattern is supportive of an usually long rs2508049-G haplotype over at least a 3.1-cM span $(\approx 7.5 \mathrm{Mb}$, from position $25.5 \mathrm{Mb}$ to $33 \mathrm{Mb}$ ).

In a previous analysis of about 3.4 million autosomal SNPs in the HapMap populations, rs2508049 showed a locusspecific branch length (an $F_{\mathrm{ST}}$-based measure of populationspecific divergence) within the top $5 \%$ of values observed in Europeans (McEvoy et al. 2006). The virtual absence of the rs2508049-G allele outside of Europe together with its extreme haplotype structure within the continent is consistent with a strong positive selective sweep in which an advantageous variant was driven to a higher frequency, dragging linked variation along with it. 

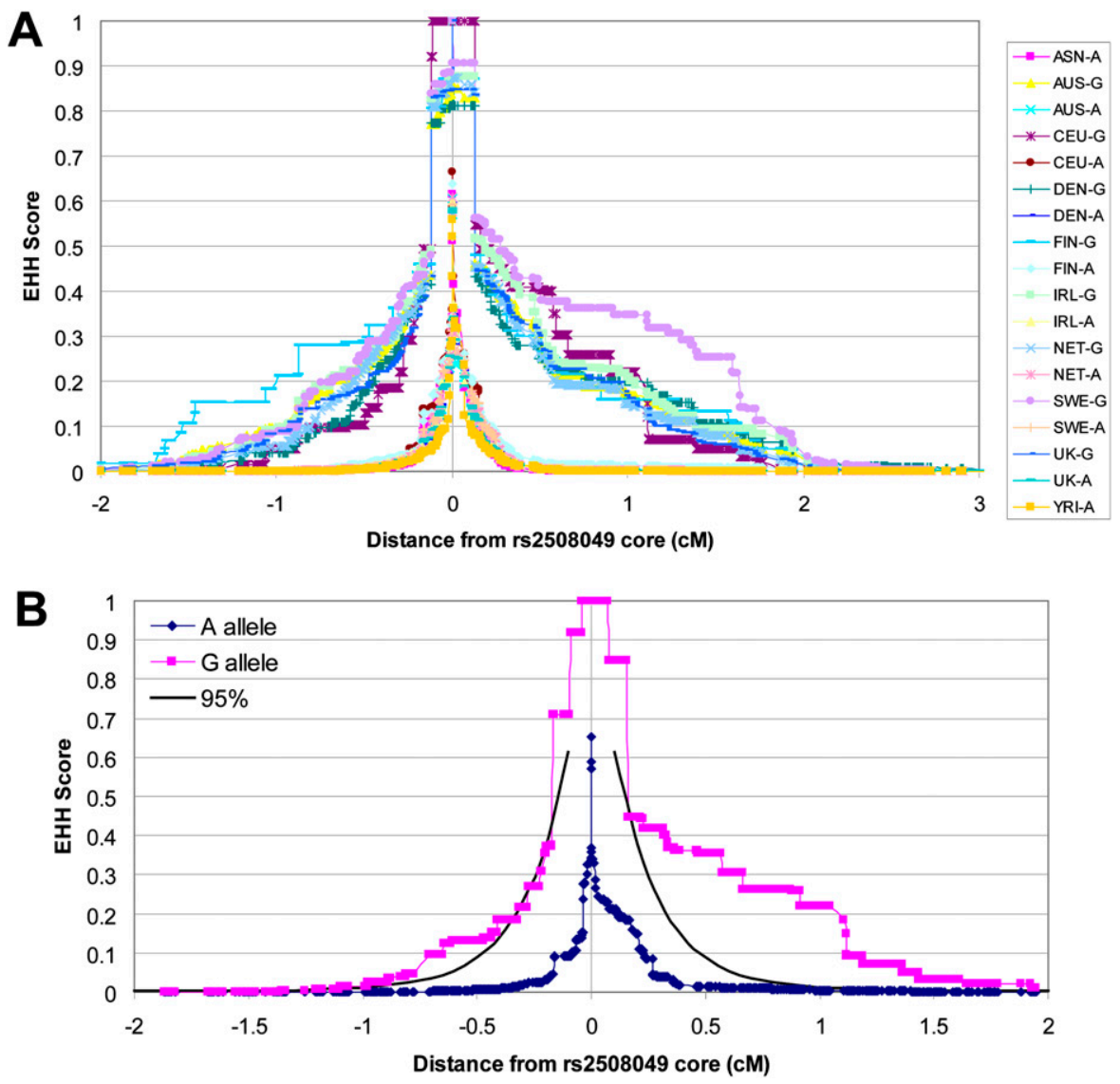

Figure 4. EHH patterns around rs 2508049 on chromosome 6. (A) EHH for the rs $2508049 \mathrm{G}$ and $\mathrm{A}$ alleles over a $5-\mathrm{cM}$ (or $\approx 9 \mathrm{Mb}$, from $25.06 \mathrm{Mb}$ to $33.93 \mathrm{Mb}$ ) region around the SNP in all Northern European populations. The EHH pattern for rs2508049-A in the HapMap Yoruban and Asian (Chinese and Japanese) is also shown, although the absence or very low frequency of the $G$ allele precludes similar analysis of it in these populations. (B) EHH for the rs2508049-A and $\mathrm{G}$ alleles using the prephased CEU HapMap data, over a 4-cM region (or $\approx 8 \mathrm{Mb}$, from $25.06 \mathrm{Mb}$ to $33.07 \mathrm{Mb}$ ). A 95 percentile boundary curve from an empirical EHH distribution of 15,325 chromosome 6 SNPs (30650 observations), calculated at $0.1-\mathrm{cM}$ intervals over a 2-cM distance from the cores, is also indicated. Population codes are as follows: (ASN) East Asian HapMap; (AUS) Australia; (CEU) European-American HapMap; (DEN) Denmark; (FIN) Finland; (IRL) Ireland; (NET) Netherlands; (SWE) Sweden; (UK) United Kingdom; (YRI) Yoruba HapMap.

\section{Discussion}

We examined whole genome polymorphism for several population samples either from, or largely descendent from, Northern Europe. In common with recent similar studies, we demonstrated that it is possible to discern clear differences among individuals from very closely related populations and even to observe stratification within the same population. The nature and relative importance/order of PCs will, of course, be affected by the populations and individuals included. Within our data, the distinctive position of Finland in PC1 may be the consequence of migrational contact with Northern Asians or admixture from the Saami population, who are known European genetic outliers. The differentiation apparent within Finland along a Northeast to Southwest axis is consistent with such a genetic diffusion.

PC2 largely separates Ireland from Britain and these from mainland Europe, and it is possible to discern further British Isles structure within this trend despite our limited information on the local ancestry of these individuals. This pattern is similar to the culmination of the Southeast to Northwest continental wide trends observed with $\mathrm{Y}$ chromosomes (Hill at al. 2000; Rosser et al. 2000; Capelli et al. 2003) and classical gene frequencies (Cavalli-Sforza et al. 1994). Although its origin is debated, it may be consistent with suggestions that Western European fringe populations, like Ireland, retain a closer genetic continuity to an earlier, perhaps pre-Neolithic European population (McEvoy et al. 2004). However, caution is warranted in interpreting these patterns since PCA trends need not be the result of a specific grand migration but can also occur under simpler demographic scenarios such as isolation by distance models (Novembre and Stephens 2008). Whatever their ultimate causes in the past, these patterns have important present-day practical ramifications in gene mapping by association. Our example of pairwise genome inflation factors between these populations is a reminder to consider structure even when combining individuals from a small and prima facie homogeneous area. As sample sizes are increased to detect associated variants of smaller effects, so the risk of confounding by even subtle stratification increases (Marchini et al. 2004).

In addition to the expected predominant role of neutral evolution (random genetic drift proportional to divergence time), and possibly migration, it appears that differential selection has had a discernable action between these Northern European populations. A previous study pioneered a similar genic/nongenic $F_{\mathrm{ST}}$ SNP test to demonstrate the role of selection between the HapMap populations (Barreiro et al. 2008). However, these populations diverged tens of thousands of years ago and have since occupied distinct geographic areas. The close relatedness of Northern Europeans and similar ecological range might suggest less opportunity for different exogenous conditions to exert a selective influence. In addition, the present study used up to 10-fold fewer SNPs than were available for the HapMap comparison, potentially weakening the power to detect such signals.

Accordingly, the general signal of positive selection in our intracontinental analysis is very modest compared to the intercontinental HapMap-based study. The top $F_{\mathrm{ST}}$ bin floor is much less here $(>0.016$ vs. $>0.85)$, for instance, and the genic fraction never exceeds the nongenic fraction in Europeans as seen with the HapMap populations. The observation that the genic enrichment among SNPs with high $F_{\mathrm{ST}}$ is largely focused on genes from a single ontology category ("immunity and defense") is support that the signal, while subtle, is real and robust. Furthermore, this category simultaneously provides a biologically plausible explanation for the detectable selection signal. Variability in the geographic extent of infectious disease outbreaks as well as potentially strong selection coefficients associated with

\section{Genome Research} www.genome.org 
such epidemics are a simple means by which selection could have a detectable effect within such a limited temporal and geographic range.

While the genic $F_{\mathrm{ST}}$ test is collective evidence for positive selection, our study identified one specific example of a probable selective event/sweep around the rs2508049-G allele in the HLA region of chromosome 6 . The exceptionally long span of haplotype homozygosity suggests relatively recent and/or intense selection across all Northern Europeans (although we cannot distinguish whether this was before or after population divergence). Unusual patterns of genetic variation within this region are well established, and it has likely been affected by a complex palimpsest of processes that seem to include positive and balancing selection (de Bakker at el. 2006; Voight et al. 2006; Sabeti et al. 2007).

It is somewhat difficult to compare homozygosity results across studies since the number of markers genotyped can influence the result. However, it appears that the rs2508049-G haplotype is one of the largest spans at appreciable frequency yet observed. To illustrate, we examined $\mathrm{EHH}$, using similar marker density and the CEU data set, around the rs4988235 SNP (also known as $-13910 \mathrm{G} / \mathrm{T}$ ) associated with the lactase (LCT) persistence trait in Europeans. The region is thought to have been intensely selected during the Neolithic period $(<10,000 \mathrm{yr}$ ago $)$ to allow the population to avail of milk and other dairy foods into adulthood (Bersaglieri et al. 2004). The like-for-like span (defined as the interval between the $5^{\prime}$ and $3^{\prime}$ points from the core where the EHH falls below 0.25) for the persistence allele, rs4988235-T $(1.3 \mathrm{Mb} / 0.4 \mathrm{cM})$, is substantially less than that observed with rs2508049-G (2 Mb/1.1 cM). However, rs4988235-T is nearly fourfold more frequent in the CEU population sample than rs2508049-G. These observations (longer span and lower allele frequency) may suggest that any selective pressure underlying the rs2508049 region was both more recent and/or of shorter duration than that at LCT.

The unusual characteristics of the rs2508049-G region are consistent with an episode of positive selection, and the pattern of haplotype decay is consistent with a target in the vicinity of rs 2508049 . The SNP is located $25 \mathrm{~kb}$ downstream from the $H L A-G$ gene, one of the HLA class I heavy chain paralogs that play a role in antigen presentation and recognition and that has also been associated with asthma (Nicolae et al. 2005), HIV susceptibility (Lajoie et al. 2006), and various pregnancy outcome phenotypes (Favier et al. 2007). Two other genes of the same family (HLA-A, $H L A-F)$ are also located close by. However, the region $( \pm 250 \mathrm{~kb})$ also encompasses loci with various other roles including a gammaaminobutyric acid neurotransmitter receptor (GABBR1). Therefore, while one selective explanation is a response to infectious disease, it is clearly not the only possible scenario. The region has also been repeatedly implicated in many disorders, many of which like asthma, multiple sclerosis, and schizophrenia, occur at a higher frequency in Europeans. While these disorders are likely to be complex, it is interesting to speculate that some of the risk may be an evolutionary hangover of selective hitchhiking.

\section{Conclusions}

Our analysis of several Northern European populations demonstrates once again the remarkable ability of dense data, in terms of both genome and population coverage, to dissect a range of events from selection to migration over a recent timeframe and across very limited geographic areas. The results also highlight the present-day legacy of the recent population past both in potential disease risk and in attempts to map complex trait loci through whole genome association.

\section{Methods}

\section{Samples and genotyping}

This study primarily used members of national twin cohorts that are part of the GenomEUtwin project (Peltonen 2003) (http:// www. GenomEUtwin.org). Genotyped individuals are one member of a monozygotic twin pair from Australia, United Kingdom, Denmark, Sweden, Netherlands, or Finland $(n=1862)$. We also included an Irish population sample used as controls in a previous case/control study of Amyotrophic lateral sclerosis (Cronin et al. 2008), as well as the European-American HapMap population (CEU) $(n=60)$. Subsets of the HapMap data for the East Asian (ASN) (Japanese + Chinese) and West African Yoruban (YRI) populations were also used for some analysis. All samples were collected with informed consent and appropriate ethical approval. The novel genotype data are deposited in the European Genotype Archive (http://www.ebi.ac.uk/ega/page.php), under the accession number EGAS00000000033, where it may be retrieved for legitimate research purposes.

Twin samples were genotyped using the Infinium II assay on the HumanHap300-Duo Genotyping Beadchips (Illumina, Inc.). Around two-thirds of the subjects were genotyped at the Finnish Genome Center (Helsinki) and the remainder at the SNP Technology Platform, Uppsala University (Uppsala, Sweden). In total, 318,237 SNPs were genotyped. There was a $99.99 \%$ reproducibility rate between SNPs in 14 duplicate samples typed at both sites. Within each center, 26 samples were genotyped in duplicate with a $99.99 \%$ consistency rate. SNPs and individuals with $>10 \%$ missing data were excluded for the purposes of the analysis described herein. The Irish sample had been typed using the Illumina Infinium II $550 \mathrm{~K}$ SNP platform. When integrating the GenomEUtwin, Irish, and HapMap samples, we included only those SNPs genotyped in all three groups where unambiguous allele matching could be made. We checked allele flipping errors in this process by screening for gross outliers in pairwise SNP $F_{\text {ST }}$ values between the GenomEUtwin and the Irish or HapMap populations. A total of 305,320 SNPs passed this process, but only those with an autosomal location were included in most analysis (296,553 SNPs).

\section{$F_{\mathrm{ST}}$ and $\delta$}

Genetic distances as $F_{\mathrm{ST}}$ values were calculated for each SNP according to Weir and Cockerham (see Weir 1996) and averaged to obtain a single estimate for each pairwise population combination or a global value over all populations. $F_{\mathrm{ST}}$ values normally range between 0 and 1, but small negative values are possible. As previously noted (Barreiro et al. 2008), this reflects the predominance of sampling error over very weak population subdivision and has no biological interpretation. $\delta$ is defined as the absolute difference in allele frequency between any two predefined groups. $F_{\mathrm{ST}}$ and $\delta$ were calculated using purpose written Perl scripts.

\section{Principal component analysis (PCA)}

We used the EIGENSOFT package (Patterson et al. 2006; Price et al. 2006), and its default parameters, to calculate up to 100 eigenvectors or principal components (PC). We first ran an exploratory analysis to identify and remove individuals who were greater than 
6 standard deviations from the mean along any of the top 10 PCs $(n=48)$. Using detailed self-reported ancestries where available (from the United Kingdom and Australian populations), it could be shown that these individuals typically had at least partial nonEuropean or Southern European ancestry. The total $(n=2051)$ cleaned sample sizes are: Australia $(n=451)$, United Kingdom $(n=433)$, Denmark $(n=161)$, Sweden $(n=302)$, Netherlands $(n=$ $284)$, Finland $(n=149)$, Ireland $(n=211)$, and CEU-HapMap $(n=$ $60)$. As a quality control measure, we tested for any significant correlation between individual scores across the top 10 PCs and individual rates of missing genotype data but observed no significant relationship.

We investigated the correspondence of PC values and geography using Mantel tests of correlation between two matrices (Mantel 1967). A matrix of geographic distances between individuals (in kilometers) was generated from generic coordinates for each European population (excluding Australia and CEU) as follows (Latitude/Longitude): Ireland: 54/-7; United Kingdom: 53/-1; Netherlands: 52/5; Denmark: 56/10; Sweden: 60/-15; Finland: 63/-27. Negative longitude values indicate Western Hemisphere locations. For each PC, a matrix of absolute interindividual differences in PC score was created. Mantel correlation significance was quantified by random permutation of matrix elements over 10,000 replicate analyses.

\section{Ancestry informative markers}

To develop and test sets of ancestry informative markers (AIMs), we divided, at random, the complete sample data set into discovery and test cohorts. The discovery data set $(n=770)$ consisted of half of each of the United Kingdom, Denmark, Sweden, Finland, Ireland, and Netherlands samples, thus preserving the relative population sample sizes of the full data set. A global $F_{\mathrm{ST}}$ value was calculated for each SNP using the discovery set and values ranked. Sets of the top 500,1500, 2500, and 5000 SNPs were selected as AIM panels with the additional proviso that no marker was within $250 \mathrm{~kb}$ of another, a measure taken to minimize LD between SNPs. These sets were used in PCA and structure analysis of the test data set. We included all the Australian and CEU-HapMap samples in the test data set $(n=1281)$ since, as admixed groups, they might skew $F_{\mathrm{ST}}$ calculations, and, secondly, we are interested in investigating their ancestry in the Northern European genetic context.

\section{structure}

In addition to PCA, we applied an alternative Bayesian clustering approach, as implemented in the program structure (v2.1), to investigate population stratification (Pritchard et al. 2000; Falush et al. 2003). The method does not rely on a priori population labels but instead infers a specified $K$ number of population clusters from the genotype data essentially using departures from HardyWeinberg equilibrium. We applied the conditions used in a similar previous analysis of European-Americans (Tian et al. 2008). Briefly, this involved using the admixture model (which allows fractional assignment of an individual's genome to different clusters) without using any population label information. To facilitate computational tractability, the entire data set $(296,553$ SNPs) was not used, but, rather, we examined the performance of each of the previously described AIM sets (500, 1500, 2500, and 5000 SNPs) in the test sample set (composed of half of the Europeans and all Australians and HapMap European-American individuals). A single run, consisting of a burn-in of 5000 replicates followed by 10,000 iterations, was carried out for each AIM set over $K=2$ to $K=5$.

\section{Extended haplotype homozygosity}

Extended haplotype homozygosity (EHH) is the probability that two randomly chosen chromosomes with the same allele at a particular core (be it a single SNP or a small haplotype) will be identical (at all genotyped SNPs) to a certain distance, either upstream or downstream, from that core (Sabeti et al. 2002). As such, it can be used to identify a selective sweep signature. In order to compute EHH scores, we first phased a large region (up to $20 \mathrm{Mb}$ ) around a core SNP of interest, for each population separately, using the fastPHASE 1.2 algorithm and its default settings (Scheet and Stephens 2006). EHH scores were then calculated using a purpose written Perl script.

$\mathrm{EHH}$ is often expressed as a ratio of the value at an allele of interest to the average at all other alleles, as a method of controlling for variation in recombination rate when comparing $\mathrm{EHH}$ scores across loci (relative $\mathrm{EHH}$ or $\mathrm{rEHH}$ ). Where the core is a single SNP, this is simply the ratio of allele A to allele B. However, once the $\mathrm{EHH}$ score for the comparative allele reaches zero, the ratio becomes undefined, and this problem increases with distance from the core. To avoid this, we work with raw EHH scores, matched for recombination distance when comparing across loci using genetic map information from the HapMap (release \#22) CEU and YRI populations.

\section{EHH significance}

There are two general approaches to gauge the significance of an observed EHH score based on either simulated or observed data. Simulation requires specification of demographic parameters (bottlenecks, expansions, etc.) that are generally unknown. Empirical data, in contrast, are the realization of these events, and loci that stand apart from the distribution of observed $\mathrm{EHH}$ values are possible targets of selection. While not a formal test of significance, we used an empirical approach to assess the "unusualness" of the rs2508049 EHH result on chromosome 6. The pre-phased genotype information for chromosome 6 in the CEU population was retrieved (Release \#22), and those alleles with a similar frequency to the initial core allele of interest were identified. Treating each of these as a core in turn, EHH was calculated at 0.1-cM intervals up to $2 \mathrm{cM}$ upstream and downstream of this position. The genotype coverage was pruned (reduced) by a factor of 12 during EHH calculations to achieve similar SNP density to that in the $305 \mathrm{~K}$ data set. The empirical distribution of the values (including two-upstream and downstream-observations per SNP) at each distance was used to construct a 95 th percentile boundary curve.

\section{Gene annotation and selection analysis}

To investigate a general signature of selection across the Northern European genome, we examined the distribution of SNP $F_{\mathrm{ST}}$ values conditioned on their location inside or outside genes. We used SNP annotation information previously compiled for Affymetrix, Illumina, and Perlegen genotyping platforms (https:// slep.unc.edu/evidence/files/README_annotations.pdf). These SNP annotations were created using the TAMAL database (Hemminger et al. 2006) based chiefly on UCSC Genome Browser files, HapMap, and dbSNP. A genic SNP was defined as one falling anywhere in the transcribed portion of a gene.

We further investigated the distribution of SNP $F_{\mathrm{ST}}$ values with respect to the function of the genes they fall in by using the PANTHER gene ontology database, which assigns many genes to one or more "biological processes" (Thomas et al. 2003). The full list of 25,431 human genes (and their genomic locations) classed

\section{Genome Research}


under 31 different ontological terms was retrieved from http:// www.pantherdb.org/. Analysis was restricted to those genes $(21,407)$ with a given autosomal map location. We used the proportion of the 296,553 autosomal SNPs in or in close proximity $( \pm 10 \mathrm{~kb})$ to genes in each ontology class as the basis for the expected value in a $\chi^{2}$ test against the numbers observed in the top $F_{\text {ST }}(>0.016)$ category.

\section{Acknowledgments}

The GenomEUtwin project is supported by the European Commission under the program "Quality of Life and Management of the Living Resources" of 5th Framework Programme (QLG2-CT2002-01254). This work was funded by the Center of Excellence in Complex Disease Genetics of the Academy of Finland (J.K., L.P., M.P., and S.R.), Nordic Center of Excellence in Disease Genetics, and The Finnish Cultural Foundation. The SNP Technology Platform in Uppsala is funded by Uppsala University and Uppsala University Hospital and the Swedish Wallenberg Foundation. We thank the staff of the SNP Platform for their contributions to genotyping. We also acknowledge financial support from the Wellcome Trust, the United Kingdom Department of Health via the National Institute for Health Research (NIHR) comprehensive Biomedical Research Centre award to Guy's and St. Thomas' NHS Foundation Trust in partnership with King's College London, Biotechnology and the Biological Sciences Research Council (BBSRC). B.P.M., A.R.M., G.W.M., and P.M.V. are supported by the National Health and Medical Research Council of Australia.

\section{References}

Barreiro, L.B., Laval, G., Quach, H., Patin, E., and Quintana-Murci, L. 2008. Natural selection has driven population differentiation in modern humans. Nat. Genet. 40: 340-345.

Bauchet, M., McEvoy, B., Pearson, L.N., Quillen, E.E., Sarkisian, T., Hovhannesyan, K., Deka, R., Bradley, D.G., and Shriver, M.D. 2007. Measuring European population stratification with microarray genotype data. Am. J. Hum. Genet. 80: 948-956.

Bersaglieri, T., Sabeti, P.C., Patterson, N., Vanderploeg, T., Schaffner, S.F., Drake, J.A., Rhodes, M., Reich, D.E., and Hirschhorn, J.N. 2004. Genetic signatures of strong recent positive selection at the lactase gene. Am. J. Hum. Genet. 74: $1111-1120$.

Capelli, C., Redhead, N., Abernethy, J.K., Gratrix, F., Wilson, J.F., Moen, T., Hervig, T., Richards, M., Stumpf, M.P., Underhill, P.A., et al. 2003. A Y chromosome census of the British Isles. Curr. Biol. 27: 979-984.

Cavalli-Sforza, L.L., Menozzi, P., and Piazza, A. 1994. The history and geography of human genes. Princeton University Press, Princeton, NJ.

Cronin, S., Berger, S., Ding, J., Schymick, J.C., Washecka, N., Hernandez, D.G., Greenway, M.J., Bradley, D.G., Traynor, B.J., and Hardiman, O. 2008. A genome-wide association study of sporadic ALS in a homogenous Irish population. Hum. Mol. Genet. 17: 768-774.

de Bakker, P.I., McVean, G., Sabeti, P.C., Miretti, M.M., Green, T., Marchini, J., Ke, X., Monsuur, A.J., Whittaker, P., Delgado, M., et al. 2006. A highresolution HLA and SNP haplotype map for disease association studies in the extended human MHC. Nat. Genet. 38: 1166-1172.

Devlin, B. and Roeder, K. 1999. Genomic control for association studies. Biometrics 55: 997-1004.

Falush, D., Stephens, M., and Pritchard, J.K. 2003. Inference of population structure using multilocus genotype data: Linked loci and correlated allele frequencies. Genetics 164: 1567-1587.

Favier, B., LeMaoult, J., Rouas-Freiss, N., Moreau, P., Menier, C., and Carosella, E.D. 2007. Research on HLA-G: An update. Tissue Antigens 69: 207-211.

Heath, S.C., Gut, I.G., Brennan, P., McKay, J.D., Bencko, V., Fabianova, E., Foretova, L., Georges, M., Janout, V., Kabesch, M., et al. 2008. Investigation of the fine structure of European populations with applications to disease association studies. Eur. J. Hum. Genet. 16: 14131429.

Hemminger, B.M., Saelim, B., and Sullivan, P.F. 2006. TAMAL: An integrated approach to choosing SNPs for genetic studies of human complex traits. Bioinformatics 22: 626-627.
Hill, E.W., Jobling, M.A., and Bradley, D.G. 2000. Y chromosome variation and Irish origins. Nature 404: 351-352.

International HapMap Consortium. 2005. A haplotype map of the human genome. Nature 437: 1299-1320.

International HapMap Consortium. 2007. A second generation human haplotype map of over 3.1 million SNPs. Nature 449: 851-861.

Lajoie, J., Hargrove, J., Zijenah, L.S., Humphrey, J.H., Ward, B.J., and Roger, M. 2006. Genetic variants in nonclassical major histocompatibility complex class I human leukocyte antigen (HLA)-E and HLA-G molecules are associated with susceptibility to heterosexual acquisition of HIV-1. J. Infect. Dis. 193: 298-301.

Lao, O., Lu, T.T., Nothnagel, M., Junge, O., Freitag-Wolf, S., Caliebe, A., Balascakova, M., Bertranpetit, J., Bindoff, L.A., Comas, D., et al. 2008. Correlation between genetic and geographic structure in Europe. Curr. Biol. 18: 1241-1248,

Mantel, N. 1967. The detection of disease clustering and a generalized regression approach. Cancer Res. 27: 209-220.

Marchini, J., Cardon, L.R., Phillips, M.S., and Donnelly, P. 2004. The effects of human population structure on large genetic association studies. Nat. Genet. 36: 512-517.

McEvoy, B., Richards, M., Forster, P., and Bradley, D.G. 2004. The Longue Durée of genetic ancestry: Multiple genetic marker systems and Celtic origins on the Atlantic facade of Europe. Am. J. Hum. Genet. 75: 693702 .

McEvoy, B., Beleza, S., and Shriver, M.D. 2006. The genetic architecture of normal variation in human pigmentation: an evolutionary perspective and model. Hum. Mol. Genet. 15: R176-R181.

Nicolae, D., Cox, N.J., Lester, L.A., Schneider, D., Tan, Z., Billstrand, C., Kuldanek, S., Donfack, J., Kogut, P., Patel, N.M., et al. 2005. Fine mapping and positional candidate studies identify HLA-G as an asthma susceptibility gene on chromosome 6p21. Am. J. Hum. Genet. 76: 349357.

Novembre, J. and Stephens, M. 2008. Interpreting principal component analyses of spatial population genetic variation. Nat. Genet. 40: $646-$ 649.

Novembre, J., Johnson, T., Bryc, K., Kutalik, Z., Boyko, A.R., Auton, A., Indap, A., King, K.S., Bergmann, S., Nelson, M.R., et al. 2008. Genes mirror geography within Europe. Nature 456: 98-101.

Patterson, N., Price, A.L., and Reich, D. 2006. Population structure and eigenanalysis. PLoS Genet. 2: e190. doi: 10.1371/journal.pgen.0020190.

Peltonen L. 2003. GenomEUtwin: A strategy to identify genetic influences on health and disease. Twin Res. 6: 354-360.

Price, A.L., Patterson, N.J., Plenge, R.M., Weinblatt, M.E., Shadick, N.A., and Reich, D. 2006. Principal components analysis corrects for stratification in genome-wide association studies. Nat. Genet. 38: 904-909.

Price, A.L., Butler, J., Patterson, N., Capelli, C., Pascali, V.L., Scarnicci, F., Ruiz-Linares, A., Groop, L., Saetta, A.A., Korkolopoulou, P., et al. 2008. Discerning the ancestry of European Americans in genetic association studies. PLoS Genet. 4: e236. doi: 10.1371/journal.pgen.0030236.

Pritchard, J.K., Stephens, M., and Donnelly, P. 2000. Inference of population structure using multilocus genotype data. Genetics 155: 945-959.

Rosser, Z.H., Zerjal, T., Hurles, M.E., Adojaan, M., Alavantic, D., Amorim, A., Amos, W., Armenteros, M., Arroyo, E., Barbujani, G., et al. 2000. Y chromosomal diversity in Europe is clinal and influenced primarily by geography, rather than by language. Am. J. Hum. Genet. 67: 1526-1543.

Sabeti, P.C., Reich, D.E., Higgins, J.M., Levine, H.Z., Richter, D.J., Schaffner, S.F., Gabriel, S.B., Platko, J.V., Patterson, N.J., and McDonald, G.J. 2002. Detecting recent positive selection in the human genome from haplotype structure. Nature 24: 832-837.

Sabeti, P.C., Varilly, P., Fry, B., Lohmueller, J., Hostetter, E., Cotsapas, C., Xie, X., Byrne, E.H., McCarroll, S.A., Gaudet, R., et al. 2007. Genome-wide detection and characterization of positive selection in human populations. Nature 449: 913-918.

Scheet, P. and Stephens, M.A. 2006. Fast and flexible statistical model for large-scale population genotype data: Applications to inferring missing genotypes and haplotypic phase. Am. J. Hum. Genet. 78: 629-644.

Seldin, M.F. and Price, A.L. 2008. Application of ancestry informative markers to association studies in European Americans. PLoS Genet. 4: e5. doi: 10.1371/journal.pgen.0040005.

Seldin, M.F., Shigeta, R., Villoslada, P., Selmi, C., Tuomilehto, J., Silva, G., Belmont, J.W., Klareskog, L., and Gregersen, P.K. 2006. European population substructure: Clustering of northern and southern populations. PLoS Genet. 15: e143. doi: 10.1371/journal.pgen.0020143.

Sturm, R.A., Duffy, D.L., Zhao, Z.Z., Leite, F.P., Stark, M.S., Hayward, N.K., Martin, N.G., and Montgomery, G.W. 2008. A single SNP in an evolutionary conserved region within intron 86 of the HERC2 gene determines human blue-brown eye color. Am. J. Hum. Genet. 82: 424431.

Thomas, P.D., Campbell, M.J., Kejariwal, A., Mi, H., Karlak, B., Daverman, R., Diemer, K., Muruganujan, A., and Narechania, A. 2003. PANTHER: A 
McEvoy et al.

library of protein families and subfamilies indexed by function. Genome Res. 13: 2129-2141.

Tian, C., Plenge, R.M., Ransom, M., Lee, A., Villoslada, P., Selmi, C. Klareskog, L., Pulver, A.E., Qi, L., Gregersen, P.K., et al. 2008. Analysis and application of European genetic substructure using $300 \mathrm{~K}$ SNP information. PLoS Genet. 4: e4. doi: 10.1371/journal.pgen.0040004.

Voight, B.F., Kudaravalli, S., Wen, X., and Pritchard, J.K. 2006. A map of recent positive selection in the human genome. PLoS Biol. 4: e72. doi: 10.1371/journal.pbio.0040072.
Weir, B.S. 1996. Genetic data analysis II, pp. 176-179. Sinauer, Sunderland, MA.

Wellcome Trust Case Control Consortium. 2007. Genome-wide association study of 14,000 cases of seven common diseases and 3000 shared controls. Nature 447: 661-678.

Received July 15, 2008; accepted in revised form February 24, 2009.

814 Genome Research 


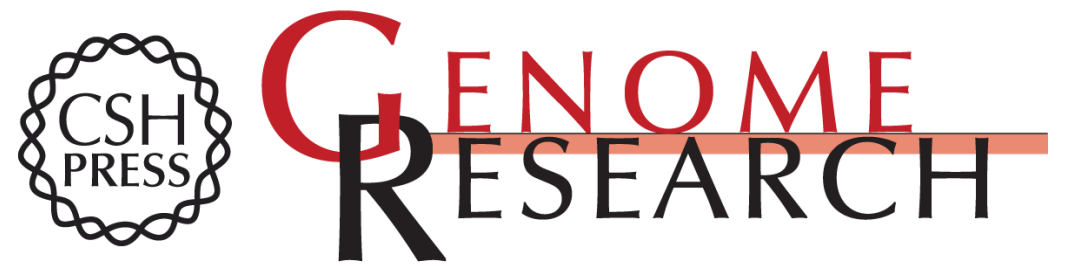

\section{Geographical structure and differential natural selection among North European populations}

Brian P. McEvoy, Grant W. Montgomery, Allan F. McRae, et al.

Genome Res. 2009 19: 804-814 originally published online March 5, 2009

Access the most recent version at doi:10.1101/gr.083394.108

\section{Supplemental http://genome.cshlp.org/content/suppl/2009/05/01/gr.083394.108.DC1 \\ Material}

Related Content Global distribution of genomic diversity underscores rich complex history of continental human populations

Adam Auton, Katarzyna Bryc, Adam R. Boyko, et al.

Genome Res. May, 2009 19: 795-803 Fine-scaled human genetic structure

revealed by SNP microarrays

Jinchuan Xing, W. Scott Watkins, David J. Witherspoon, et al.

Genome Res. May , 2009 19: 815-825 Non-Darwinian estimation: My ancestors, my genes' ancestors

Kenneth M. Weiss and Jeffrey C. Long

Genome Res. May, 2009 19: 703-710

References This article cites 39 articles, 4 of which can be accessed free at:

http://genome.cshlp.org/content/19/5/804.full.html\#ref-list-1

Articles cited in:

http://genome.cshlp.org/content/19/5/804.full.html\#related-urls

\section{License}

Email Alerting Receive free email alerts when new articles cite this article - sign up in the box at the Service top right corner of the article or click here.

\section{Affordable, Accurate Sequencing.}

To subscribe to Genome Research go to:

https://genome.cshlp.org/subscriptions 